\title{
On the well-posedness of generalized Darcy-Forchheimer equation
}

\author{
Johnson D. Audu ${ }^{1 *}$, Faisal A. Fairag ${ }^{1}$ and Salim A. Messaoudi ${ }^{1}$
}

\section{"Correspondence:}

jdaudy@kfupm.edu.sa

1 Department of Mathematics and

Statistics, King Fahd University of

Petroleum and Minerals, Dhahran,

Saudi Arabia

\begin{abstract}
Under the necessary compatibility condition and some mild regularity assumptions on the interior and the boundary data, we prove the existence, uniqueness, and stability of solution in $\left[L^{m+1}(\Omega)\right]^{d} \times\left(W^{1, \frac{m+1}{m}}(\Omega) \cap L_{0}^{2}(\Omega)\right)$ for a generalized Darcy-Forchheimer model, governing a non-Darcy flows in porous media with dimension $d=2,3$ and $m \in(1,2]$.

Keywords: Porous media; Darcy-Forchheimer; Monotone-type; Existence; Stability
\end{abstract}

\section{Introduction}

The applications of nonlinear differential equations are seen in many fields (see [1-4]). In the field of fluid dynamics, the nonlinear correction to Darcy's law has been an active area of research for many years. Theoretical, experimental, and numerical analyses [5-7] have been performed to ascertain the exact form and magnitude of the nonlinearity effect. However, until now, no single correction seems to be acceptable by all (see [7]). Non-Darcy effects are prevalent in fluid transport through porous media, especially for high velocity flows $[8,9]$. Worthy of note includes Darcy-Forchheimer which serves as a mathematical model for many high velocity flows in porous media, most especially for gas reservoirs and hydrodynamic flows [10-12]. The generalized nonlinear Darcy-Forchheimer equation takes the form

$$
G(\rho, \boldsymbol{u})+\nabla p=\boldsymbol{f} \quad \text { in } \Omega
$$

In (1), $p$ is the pressure, $\boldsymbol{u}$ and $\rho$ are the velocity and density of the fluid, respectively, $\boldsymbol{f}$ is a vector function, usually the gradient of the depth function. Different forms of (1) have been considered by several researchers, see, for example, [10-15]. Forchheimer in his work [5] established three empirical nonlinear formulas: $-\nabla p=\alpha \boldsymbol{v}+\beta|\boldsymbol{v}| \boldsymbol{v},-\nabla p=$ $\alpha \boldsymbol{v}+\beta|\boldsymbol{v}| \boldsymbol{v}+\gamma|\boldsymbol{v}|^{2} \mathbf{v}$, and $-\nabla p=\alpha \boldsymbol{v}+\beta|\boldsymbol{v}|^{m-1} \mathbf{v}, m \in(1,2)$, to account for the inadequacies of Darcy law with $\alpha, \beta$, and $\gamma$ empirical constants deduced from experimental data. The model of interest in this article is the following generalized flow law:

$$
\frac{\mu}{\rho} \boldsymbol{K}^{-1} \boldsymbol{u}+\frac{\beta}{\rho}|\boldsymbol{u}|^{m-1} \boldsymbol{u}+\nabla p=\boldsymbol{f} \quad \text { in } \Omega, m \in(1,2],
$$

(c) The Author(s) 2018. This article is distributed under the terms of the Creative Commons Attribution 4.0 International License (http://creativecommons.org/licenses/by/4.0/), which permits unrestricted use, distribution, and reproduction in any medium, provided you give appropriate credit to the original author(s) and the source, provide a link to the Creative Commons license, and indicate if changes were made. 
where $\Omega$ is an open bounded domain of $\mathbb{R}^{d}(d=2$ or 3 ) with a Lipschitz boundary $\Gamma$, $\boldsymbol{u}: \Omega \longrightarrow \mathbb{R}^{d}$ and $p: \Omega \longrightarrow \mathbb{R}$ denote the unknown velocity vector and scalar pressure field, respectively, and $\boldsymbol{f}$ is a given function. The permeability tensor is assumed to be uniformly positive definite and bounded, while the parameters $\mu, \rho$, and $\beta$ are assumed to be constants. We denote by $|\cdot|$ the Euclidean norm such that $|\boldsymbol{u}|^{m-1}=(\boldsymbol{u} \cdot \boldsymbol{u})^{\frac{m-1}{2}}$. For $m=2$, Equation (2) reduces to the classical Darcy-Forchheimer law $[5,16,17]$.

We solve (2) subject to the following conditions:

$$
\operatorname{div} \boldsymbol{u}=b \quad \text { in } \Omega \quad \text { and } \quad \boldsymbol{u} \cdot \boldsymbol{n}=g \quad \text { on } \Gamma .
$$

In (3), $b$ and $g$ are the interior and boundary data, respectively, satisfying the compatibility condition

$$
\int_{\Omega} b(x) d x=\int_{\Gamma} g(\sigma) d \sigma .
$$

Equation (2)-(4) is a reliable model for describing a single-phase strong inertia flow [18, 19] in simple and complex porous media.

In this article, we establish the well-posedness of (2)-(4). Our technique of proof is similar to the one in [20] with some necessary modifications due to the nature of the nonlinear term. We now state our main result, which will be proved by a combination of classical arguments for saddle point problems [21, 22] and monotone nonlinear elliptic problems $[23,24]$.

Theorem 1.1 Provided $b \in L^{\frac{d(m+1)}{d+(m+1)}}(\Omega)$ and $g \in L^{\frac{(d-1)(m+1)}{d}}(\Gamma)$ satisfy (4), Problem (2)-(4) has a unique solution $(\boldsymbol{u}, p)$, with $\boldsymbol{u} \in\left[L^{m+1}(\Omega)\right]^{d}$ and $p \in W^{1, \frac{m+1}{m}}(\Omega) \cap L_{0}^{2}(\Omega)$.

The remainder of the article is organized as follows: Some preliminaries are presented in Sect. 2. The variational formulation will be given in Sect. 3. We prove the existence and uniqueness of solution in Sect. 4, while the stability is established in Sect. 5.

\section{Preliminaries}

This section is devoted to recalling some notations, definitions, and some classical results. Let $\Omega$ be a measurable subset of $\mathbb{R}^{d}$,

$$
L^{p}(\Omega)=\left\{u: \Omega \longrightarrow \mathbb{R} \text { is measurable and } \int_{\Omega}|u|^{p} d x<\infty, 1 \leq p<\infty\right\} .
$$

The $L^{p}$ norm of a function $f$ is given by

$$
\|u\|_{L^{p}(\Omega)}=\left(\int_{\Omega}|u|^{p}\right)^{\frac{1}{p}} .
$$

For a given domain $\Omega$ and for any $k \in \mathbb{N}$, let $\|\cdot\|_{W^{k, p}(\Omega)}$ and $|\cdot|_{W^{k, p}(\Omega)}, 1 \leq p<\infty$, be the norm and semi-norm, respectively, on the standard Sobolev space $W^{k, p}(\Omega)$ (see [25]). For a vector-valued function $\boldsymbol{v}=\left(v_{1}, v_{2}, \ldots, v_{d}\right) \in\left[W^{k, p}(\Omega)\right]^{d}$, the norm

$$
\|\boldsymbol{v}\|_{\left[W^{k, p}(\Omega)\right]^{d}}=\left(\sum_{i=1}^{d}\left\|\boldsymbol{v}_{i}\right\|_{W^{k, p}(\Omega)}^{p}\right)^{\frac{1}{p}} .
$$


Similarly, the semi-norm is given by

$$
|\boldsymbol{V}|_{\left[W^{k, p}(\Omega)\right]^{d}}=\left(\sum_{i=1}^{d}\left|\boldsymbol{v}_{i}\right|_{W^{k, p}(\Omega)}^{p}\right)^{\frac{1}{p}} .
$$

Note that $\|\cdot\|_{\left[W^{k, p}(\Omega)\right]^{d}}$ reduces to $\|\cdot\|_{\left[L^{p}(\Omega)\right]^{d}}$ when $k=0$.

The following inequalities will be useful in our calculations and can be found in [24, 25]:

$$
\begin{aligned}
& (a+b)^{p} \leq 2^{p-1}\left(a^{p}+b^{p}\right), \quad \forall a, b \in[0, \infty), p \in(1, \infty), \\
& a b \leq \frac{a^{p}}{p}+\frac{b^{q}}{q}, \quad a, b \in[0, \infty), \quad \frac{1}{p}+\frac{1}{q}=1, \quad p, q \in(0, \infty) .
\end{aligned}
$$

Lemma $2.1([26])$ Let $\left(X,\|\cdot\|_{X}\right)$ and $\left(M,\|\cdot\|_{M}\right)$ be two reflexive Banach spaces. Let $\left(X^{*}\right.$, $\left.\|\cdot\|_{X^{*}}\right),\left(M^{*},\|\cdot\|_{M^{*}}\right)$ be their corresponding duals. Let $\mathcal{B}: X \longrightarrow M^{*}$ be a linear continuous operator and $\mathcal{B}^{*}: M \longrightarrow X^{*}$ be the dual of $\mathcal{B}$. Let $V=\operatorname{ker}(\mathcal{B})$ be a kernel of $\mathcal{B}$. Denote by $V^{0} \subset X$ the polar subspace of $V, V^{0}=\left\{x^{*} \in X^{*} \mid\left\langle x^{*}, v\right\rangle=0, \forall v \in X\right\}$ and $\dot{\mathcal{B}}: X / V \longrightarrow M^{*}$ the quotient operator associated with $\mathcal{B}$. Then the following properties are equivalent:

(i) There exists a constant $\alpha>0$ such that

$$
\inf _{q \in M \backslash\{0\}} \sup _{u \in X \backslash\{0\}} \frac{\langle\mathcal{B} u, q\rangle}{\|q\|_{M}\|u\|_{X}} \geq \alpha ;
$$

(ii) $\mathcal{B}^{*}$ is an isomorphism from $M$ onto $V^{0}$ and

$$
\left\|\mathcal{B}^{*} q\right\|_{X^{*}} \geq \alpha\|q\|_{M}, \quad \forall q \in M
$$

(iii) $\dot{\mathcal{B}}$ is an isomorphism from $X / V$ onto $M^{*}$ and

$$
\|\dot{M} \dot{u}\|_{M^{*}} \geq \alpha\|\dot{u}\|_{X / V}, \quad \forall \dot{u} \in X / V
$$

\section{Variational formulation}

We define the following spaces:

$$
\begin{aligned}
& L_{0}^{2}(\Omega)=\left\{v: v \in L^{2}(\Omega), \int_{\Omega} v(x) d x=0\right\}, \\
& X=\left[L^{m+1}(\Omega)\right]^{d}, \\
& M=W^{1, \frac{m+1}{m}}(\Omega) \cap L_{0}^{2}(\Omega) .
\end{aligned}
$$

The zero mean condition is required to guarantee the uniqueness of the pressure $p$. We consider the following variational formulation:

For any $\boldsymbol{f} \in X^{*}$, find a pair of functions $(\boldsymbol{u}, p) \in X \times M$ such that

$$
\begin{aligned}
& \frac{\mu}{\rho} \int_{\Omega}\left(K^{-1} \boldsymbol{u}\right) \cdot \boldsymbol{\varphi} d x+\frac{\beta}{\rho} \int_{\Omega}|\boldsymbol{u}|^{m-1} \boldsymbol{u} \cdot \boldsymbol{\varphi} d \mathbf{x}+\int_{\Omega} \nabla p \cdot \boldsymbol{\varphi} d \mathbf{x}=\int_{\Omega} \boldsymbol{f} \cdot \boldsymbol{\varphi} d \mathbf{x} \\
& \int_{\Omega} \nabla q \cdot \mathbf{u} d \mathbf{x}=-\int_{\Omega} b q \boldsymbol{d} x+\int_{\Gamma} g q \boldsymbol{d} \sigma
\end{aligned}
$$

$\forall \varphi \in X, \forall q \in M$, with $b \in L^{\frac{d(m+1)}{d+(m+1)}}(\Omega)$ and $g \in L^{\frac{(d-1)(m+1)}{d}}(\Gamma)$ satisfying (4). 
Problems (12)-(13) and (2)-(4) are equivalent. To see this, we multiply equation (2) by $\varphi \in X$ and integrate over $\Omega$. We then apply the conditions in equation (3) to the Green's formula (14):

$$
\int_{\Omega} \boldsymbol{v} \cdot \nabla q d \boldsymbol{x}=-\int_{\Omega} q \operatorname{div} \mathbf{v} d \mathbf{x}+\langle q, \boldsymbol{v} \cdot n\rangle_{\Gamma}, \quad \forall q \in M, \forall \boldsymbol{v} \in H
$$

with

$$
H=\left\{\boldsymbol{v} \in\left[L^{m+1}(\Omega)\right]^{d} ; \operatorname{div} \boldsymbol{v} \in L^{\frac{d(m+1)}{d+(m+1)}}(\Omega)\right\}
$$

Define the following operators:

$$
\begin{aligned}
& \mathcal{A}: X \rightarrow X^{*}, \quad\langle\mathcal{A}(\boldsymbol{u}), \varphi\rangle_{X^{*} \times X}:=a(\boldsymbol{u}, \boldsymbol{\varphi}), \quad \forall \boldsymbol{\varphi} \in X, \\
& \boldsymbol{B}: X \rightarrow M^{*}, \quad\langle\mathbf{B u}, q\rangle_{M^{*} \times M}:=b(\boldsymbol{u}, q), \quad q \in M, \\
& \boldsymbol{B}^{*}: M \rightarrow X^{*}, \quad\left\langle\boldsymbol{B}^{*}(p), \varphi\right\rangle_{X^{*} \times X}:=b(\boldsymbol{\varphi}, p), \quad \boldsymbol{\varphi} \in X .
\end{aligned}
$$

With these operators, an equivalent form of (12)-(13) is as follows:

Given $(\boldsymbol{f}, g) \in X^{*} \times M^{*}$, we want to find a pair $(\boldsymbol{u}, p) \in X \times M$ such that

$$
\begin{cases}a(\boldsymbol{u}, \boldsymbol{\varphi})+b(\boldsymbol{\varphi}, p)=\langle\boldsymbol{f}, \boldsymbol{\varphi}\rangle_{X^{*}, X}, & \forall \boldsymbol{\varphi} \in X \\ b(\boldsymbol{u}, q)=\langle g, q\rangle_{M^{*} \times M}, & \forall q \in M\end{cases}
$$

Problem (15) can also be rewritten as

$$
\begin{cases}\mathcal{A}(\boldsymbol{u})+\boldsymbol{B}^{*}(p)=\boldsymbol{f} & \text { in } X^{*} \\ \boldsymbol{B} u=g & \text { in } M^{*}\end{cases}
$$

where

$$
\begin{aligned}
& \mathcal{A}(\boldsymbol{u})=\frac{\mu}{\rho} \boldsymbol{K}^{-1} \boldsymbol{u}+\frac{\beta}{\rho}|\boldsymbol{u}|^{m-1} \boldsymbol{u}, \\
& \langle\boldsymbol{B} \boldsymbol{u}, q\rangle=\int_{\Omega} \nabla q \cdot \boldsymbol{u} d \mathbf{x}, \\
& \left\langle\boldsymbol{B}^{*}(p), \boldsymbol{u}\right\rangle=\int_{\Omega} \nabla p \cdot \boldsymbol{u} d \mathbf{x}, \\
& F(q)=\langle g, q\rangle_{M^{*} \times M}=-\int_{\Omega} b q \boldsymbol{d} x+\int_{\Gamma} g q \boldsymbol{d} \sigma .
\end{aligned}
$$

In the sequel, Problems (15) and (16) are used interchangeably just for convenience.

Lemma 3.1 The following inf-sup condition holds:

$$
\inf _{q \in M \backslash\{0\}} \sup _{\boldsymbol{u} \in X \backslash\{0\}} \frac{b(\boldsymbol{u}, q)}{\|q\|_{M}\|\boldsymbol{u}\|_{X}}=1 .
$$


Proof By representation of a dual norm,

$$
\|\boldsymbol{v}\|_{\left[L^{\frac{m+1}{m}}(\Omega)\right]^{d}}=\sup _{\boldsymbol{u} \in\left[L^{m+1}(\Omega)\right]^{d}} \frac{\int_{\Omega} \boldsymbol{v} \cdot \boldsymbol{u} d \mathbf{x}}{\|\boldsymbol{u}\|_{\left[L^{m+1}(\Omega)\right]^{d}}}, \quad \forall \boldsymbol{v} \in\left[L^{\frac{m+1}{m}}(\Omega)\right]^{d}
$$

By setting $\boldsymbol{v}=\nabla q$, we get

$$
\|\nabla q\|_{\left[L^{\frac{m+1}{m}}(\Omega)\right]^{d}}=\sup _{\|\boldsymbol{u}\|_{\left[L^{m+1}(\Omega)\right]^{d}}} \frac{\int_{\Omega} \nabla q \cdot \mathbf{u} d \mathbf{x}}{\|u\|_{\left[L^{m+1}(\Omega)\right]^{d}}}, \quad \forall q \in M .
$$

Since $q$ belongs to the space of zero mean, we have the following:

$$
\|\nabla q\|_{\left[L^{\frac{m+1}{m}}(\Omega)\right]^{d}}=\|q\|_{M}, \quad \forall q \in M .
$$

Equivalently,

$$
\inf _{q \in M \backslash\{0\}} \frac{\|\nabla q\|_{\left[L^{\frac{m+1}{m}}(\Omega)\right]^{d}}}{\|q\|_{M}}=1 .
$$

Now substituting (23) in (24) yields the required result.

Proposition 3.1 For each $b \in L^{\frac{d(m+1)}{d+(m+1)}}(\Omega)$ and $g \in L^{\frac{(d-1)(m+1)}{d}}(\Gamma)$ satisfying (4), there is unique $\mathbf{u}_{\ell} \in\left[L^{m+1}(\Omega)\right]^{d / V}$ satisfying

$$
\int_{\Omega} \mathbf{u}_{\ell} \cdot \nabla q d \mathbf{x}=-\int_{\Omega} b q d \mathbf{x}+\int_{\Gamma} g q d \sigma, \quad \forall q \in M
$$

Furthermore,

$$
\left\|\boldsymbol{u}_{\ell}\right\|_{L^{m+1 / V}} \leq C\left(\|b\|_{L^{\frac{d(m+1)}{d+(m+1)}(\Omega)}}+\|g\|_{L^{\frac{(d-1)(m+1)}{d}(\Gamma)}}\right)
$$

where $C$ is a constant depending on $\Omega$ only.

Proof Denote

$$
\begin{aligned}
V & :=\operatorname{Ker} \boldsymbol{B}=\{\boldsymbol{u} \in X, \boldsymbol{B} \boldsymbol{u}=0\} \\
& =\left\{\boldsymbol{u} \in X, b(\boldsymbol{u}, q)=\int_{\Omega} \nabla q \cdot \boldsymbol{u} d \boldsymbol{x}=0, \forall q \in M\right\} .
\end{aligned}
$$

Equation $(15)_{2}$ can be written as follows:

$$
b(\boldsymbol{u}, q)=F(q), \quad \forall q \in M,
$$

where $F(q)=-\int_{\Omega} b q \boldsymbol{d} x+\int_{\Gamma} g q \boldsymbol{d} \sigma$. Now let us estimate the right-hand side of (28).

$$
\begin{aligned}
& |F(q)|=\left|-\int_{\Omega} b q d \mathbf{x}+\int_{\Gamma} g q d \sigma\right| \\
& \leq\|b\|_{L^{\frac{d(m+1)}{d+(m+1)}(\Omega)}}\|q\|_{L^{\frac{d(m+1)}{m(d-1)-1}(\Omega)}}+\|g\|_{L^{\frac{(d-1)(m+1)}{d}}(\Gamma)}\|q\|_{L^{\frac{(d-1)(m+1)}{m(d-1)-1}}(\Gamma)} .
\end{aligned}
$$


This implies

$$
|F(q)| \leq\left(\|b\|_{L^{\frac{d(m+1)}{d+(m+1)}(\Omega)}}+\|g\|_{L^{\frac{(d-1)(m+1)}{d}}(\Gamma)}\right)\left(\|q\|_{L^{\frac{d(m+1)}{m(d-1)-1}(\Omega)}}+\|q\|_{L^{\frac{(d-1)(m+1)}{m(d-1)-1}}(\Gamma)}\right) .
$$

By the trace theorem and Sobolev embeddings [25], we obtain

$$
\begin{aligned}
& |F(q)| \\
& \leq\left(\|b\|_{L^{\frac{d(m+1)}{d+(m+1)}(\Omega)}}+\|g\|_{L^{\frac{(d-1)(m+1)}{d}}(\Gamma)}\right)\left(c_{1}\|q\|_{W^{1}, \frac{m+1}{m}(\Omega)}+c_{2}\|q\|_{W^{1, \frac{m+1}{m}}(\Omega)}\right) \\
& \leq C\left(\|b\|_{L^{\frac{d(m+1)}{d+(m+1)}(\Omega)}}+\|g\|_{L^{\frac{(d-1)(m+1)}{d}}(\Gamma)}\right)\|q\|_{W^{1}, \frac{m+1}{m}(\Omega)} .
\end{aligned}
$$

The map $q \mapsto-\int_{\Omega} b q d \mathbf{x}+\int_{\Gamma} g q d \sigma$ is a bounded linear map, so it belongs to $M^{*}$. Therefore, using the inf-sup condition in Lemma (3.1) and the equivalence statements in Lemma 2.1, there is unique $\boldsymbol{u}_{\ell} \in\left[L^{m+1}(\Omega)\right]^{d} / V$ satisfying (25). Hence,

$$
b\left(\boldsymbol{u}_{\ell}, q\right) \leq C\left(\|b\|_{L^{\frac{d(m+1)}{d+(m+1)}(\Omega)}}+\|g\|_{L}^{\frac{(d-1)(m+1)}{d}}(\Gamma)\right]\|\nabla q\|_{\left[L^{\frac{m+1}{m}}(\Omega)\right]^{d}} .
$$

Applying Lemma 3.1 in (32), (26) is established.

Based on Proposition 3.1, we split the solution $\boldsymbol{u}$ into $\boldsymbol{u}_{0}+\boldsymbol{u}_{\ell}$, where $\boldsymbol{u}_{0} \in V$ and $\boldsymbol{u}_{\ell} \in$ $\left[L^{m+1}(\Omega)\right]^{d}$. An equivalence variational formulation of (12)-(13) is as follows: For any $f \in$ $X^{*}$, find $\boldsymbol{u}_{0} \in V$ such that

$$
\int_{\Omega} \mathcal{A}\left(\boldsymbol{u}_{0}+\boldsymbol{u}_{\ell}\right) \cdot \boldsymbol{\varphi} d \mathbf{x}=\int_{\Omega} \boldsymbol{f} \cdot \boldsymbol{\varphi} d \mathbf{x}, \quad \forall \boldsymbol{\varphi} \in V .
$$

Proposition 3.2 Problem (12)-(13) is equivalent to Problem (33).

Proof Suppose $(\boldsymbol{u}, p)$, with $\boldsymbol{u} \in X, p \in M$, is a solution of (12)-(13). Then we write $\boldsymbol{u}=$ $\boldsymbol{u}_{0}+\boldsymbol{u}_{\ell}$, where $\boldsymbol{u}_{\ell} \in\left[L^{m+1}(\Omega)\right]^{d} / V$ solves (26). It follows that $\boldsymbol{u}_{0}$ satisfies (33).

Conversely, take $\boldsymbol{u}_{0}$ to be a solution of (33); then

$$
\int_{\Omega}\left(\boldsymbol{f}-\mathcal{A}\left(\boldsymbol{u}_{0}+\boldsymbol{u}_{\ell}\right)\right) \cdot \boldsymbol{\varphi} d \mathbf{x}=0, \quad \forall \boldsymbol{\varphi} \in V
$$

Equivalently, $\boldsymbol{f}-\mathcal{A}\left(\boldsymbol{u}_{0}+\boldsymbol{u}_{\ell}\right) \in V^{0}$, where

$$
V^{0}=\left\{\boldsymbol{v} \in\left[L^{\frac{m+1}{m}}(\Omega)\right]^{d} ; \forall \boldsymbol{w} \in V, \int_{\Omega} \boldsymbol{v} \cdot \boldsymbol{w} d \mathbf{x}=0\right\}=(\operatorname{Ker} B)^{0} .
$$

It follows from the closed range theorem [27] that $\boldsymbol{f}-\mathcal{A}\left(\boldsymbol{u}_{0}+\boldsymbol{u}_{\ell}\right) \in \operatorname{Image}\left(\boldsymbol{B}^{*}\right)$.

Thanks to the inf-sup condition in Lemma 3.1 and the isomorphism in Lemma 2.1, there exists unique $p \in M$ such that

$$
\boldsymbol{B}^{*} p=\boldsymbol{f}-\mathcal{A}\left(\boldsymbol{u}_{0}+\boldsymbol{u}_{\ell}\right)
$$

Consequently,

$$
\nabla p=\boldsymbol{f}-\mathcal{A}\left(\boldsymbol{u}_{0}+\boldsymbol{u}_{\ell}\right)
$$


Hence,

$$
\frac{\mu}{\rho} \int_{\Omega} \mathcal{A}\left(\boldsymbol{u}_{0}+\boldsymbol{u}_{\ell}\right) \cdot \boldsymbol{\varphi} d \mathbf{x}+\int_{\Omega} \nabla p \cdot \boldsymbol{\varphi} d \mathbf{x}=\int_{\Omega} \boldsymbol{f} \cdot \boldsymbol{\varphi} d \mathbf{x}, \quad \forall \boldsymbol{\varphi} \in X .
$$

Since $\boldsymbol{u}=\boldsymbol{u}_{\mathbf{0}}+\boldsymbol{u}_{\ell},(12)$ is satisfied. Furthermore, since $\boldsymbol{u}_{0} \in V$, we have

$$
\begin{aligned}
\boldsymbol{B} \boldsymbol{u} & =\boldsymbol{B} \boldsymbol{u}_{0}+\boldsymbol{B} \boldsymbol{u}_{\ell} \\
& =\boldsymbol{B} \boldsymbol{u}_{\ell}=g .
\end{aligned}
$$

This implies that (13) is satisfied.

In view of the equivalence in Proposition (3.2), Problem (33) will be the focus of our analysis.

Lemma 3.2 For any pair $(\boldsymbol{v}, \boldsymbol{w}) \in \mathbb{R}^{n} \times \mathbb{R}^{n}$,

$$
\left.|| \boldsymbol{v}\right|^{m-1} \boldsymbol{v}-|\boldsymbol{w}|^{m-1} \boldsymbol{w}\left|\leq m 2^{m-2}\left[|\boldsymbol{v}|^{m-1}+|\boldsymbol{w}|^{m-1}\right]\right| v-w \mid, \quad m \geq 1 .
$$

Proof Let $(\boldsymbol{v}, \boldsymbol{w}) \in \mathbb{R}^{n} \times \mathbb{R}^{n}, \psi(\boldsymbol{v}):=|\boldsymbol{v}|^{m-1} \boldsymbol{v}, \psi(\boldsymbol{w}):=|\boldsymbol{w}|^{m-1} \boldsymbol{w}$.

For $n=2$, set

$$
\psi(\boldsymbol{v})=\left[\begin{array}{c}
\psi_{1}(v) \\
\left.\psi_{2}(v)\right)
\end{array}\right]=\left[\begin{array}{c}
|\boldsymbol{v}|^{m-1} v_{1} \\
|\boldsymbol{v}|^{m-1} v_{2}
\end{array}\right]
$$

It follows that

$$
\begin{aligned}
& \psi^{\prime}(\boldsymbol{v})=\left[\begin{array}{ll}
\frac{\partial \psi_{1}(v)}{\partial v_{1}} & \frac{\partial \psi_{1}(v)}{\partial v_{2}} \\
\frac{\partial \psi_{2}(v)}{\partial v_{1}} & \frac{\partial \psi_{2}(v)}{\partial v_{2}}
\end{array}\right], \\
& \psi^{\prime}(\boldsymbol{v})=\left[\begin{array}{cc}
(m-1)|\boldsymbol{v}|^{m-3} v_{1}^{2}+|\boldsymbol{v}|^{m-1} & (m-1)|\boldsymbol{v}|^{m-3} v_{1} v_{2} \\
(m-1)|\boldsymbol{v}|^{m-3} v_{1} v_{2} & (m-1)|\boldsymbol{v}|^{m-3} v_{2}^{2}+|\boldsymbol{v}|^{m-1}
\end{array}\right] .
\end{aligned}
$$

The Jacobian matrix $\psi^{\prime}(\boldsymbol{v})$ is $2 \times 2$ symmetric, so its norm $\left\|\psi^{\prime}(\boldsymbol{v})\right\|$ is the larger eigenvalue (spectral radius). The eigenvalues are computed by the following formula:

$$
\lambda_{1,2}=\frac{1}{2}\left[\left(a_{11}+a_{22}\right) \pm \sqrt{4 a_{12} a_{21}+\left(a_{11}-a_{22}\right)^{2}}\right] .
$$

We have

$$
\begin{aligned}
\sqrt{4 a_{12} a_{21}+\left(a_{11}-a_{22}\right)^{2}} & =\sqrt{4(m-1)^{2}|\boldsymbol{v}|^{2(m-3)} v_{1}^{2} v_{2}^{2}+(m-1)^{2}|\boldsymbol{v}|^{2(m-3)}\left(v_{1}^{2}-v_{2}^{2}\right)^{2}} \\
& =(m-1)|\boldsymbol{v}|^{(m-3)} \sqrt{4 v_{1}^{2} v_{2}^{2}+\left(v_{1}^{2}-v_{2}^{2}\right)^{2}} \\
& =(m-1)|\boldsymbol{v}|^{(m-3)} \sqrt{\left(v_{1}^{2}+v_{2}^{2}\right)^{2}} \\
& =(m-1)|\boldsymbol{v}|^{(m-3)}|\boldsymbol{v}|^{2} \\
& =(m-1)|\boldsymbol{v}|^{m-1} .
\end{aligned}
$$

Then $\lambda_{1,2}=\frac{1}{2}\left[(m-1)|\boldsymbol{v}|^{m-1}+2|\boldsymbol{v}|^{m-1} \pm(m-1)|\boldsymbol{v}|^{m-1}\right]$. 
The larger eigenvalue is given by

$$
\lambda_{1}=\frac{1}{2}\left[(m-1)|\boldsymbol{v}|^{m-1}+2|\mathbf{v}|^{m-1}+(m-1)|\mathbf{v}|^{m-1}\right]=m|\mathbf{v}|^{m-1} .
$$

Therefore, the norm of the Jacobian matrix is given by $\left\|\psi^{\prime}(\boldsymbol{v})\right\|=m|\mathbf{v}|^{m-1}$.

Application of the mean value theorem for vector-valued functions [28] yields

$$
\begin{aligned}
|\psi(\boldsymbol{v})-\psi(\boldsymbol{w})| & \leq \sup _{0 \leq t \leq 1}\|\psi \prime(\boldsymbol{w}+t(\boldsymbol{v}-\boldsymbol{w}))\||\boldsymbol{v}-\boldsymbol{w}| \\
& \leq \sup _{0 \leq t \leq 1} m|\boldsymbol{w}+t(\boldsymbol{v}-\boldsymbol{w})|^{m-1}|\boldsymbol{v}-\boldsymbol{w}| \\
& \leq m \sup _{0 \leq t \leq 1}[|\boldsymbol{w}(1-t)|+t|\boldsymbol{v}|]^{m-1}|\boldsymbol{v}-\boldsymbol{w}| \\
& \leq m[|\boldsymbol{w}|+|\boldsymbol{v}|]^{m-1}|\boldsymbol{v}-\boldsymbol{w}| .
\end{aligned}
$$

So, in view of inequality (7), we obtain

$$
|\psi(\boldsymbol{v})-\psi(\boldsymbol{w})| \leq m 2^{m-2}\left[|\boldsymbol{v}|^{m-1}+|\boldsymbol{w}|^{m-1}\right]|\boldsymbol{v}-\boldsymbol{w}|
$$

\section{Existence and uniqueness}

To prove the existence and uniqueness of solution for (33), it suffices to demonstrate that the map $\mathcal{A}$ defined in (17) satisfies the following properties in $\left[L^{m+1}(\Omega)\right]^{d}$ : boundedness, strict monotonicity, coercivity, and hemi-continuity [23, 24]. We let the least eigenvalue of $K$ be $\lambda_{s}$. Therefore,

$$
K(\boldsymbol{x}) \boldsymbol{u} \cdot \boldsymbol{u} \geq \lambda_{s}|\boldsymbol{u}|^{2}, \quad \text { for all } \boldsymbol{u} \in \mathbb{R}^{d} \text { and for all } \boldsymbol{x} \in \Omega
$$

Lemma 4.1 The operator $\mathcal{A}:\left[L^{m+1}(\Omega)\right]^{d} \rightarrow\left[L^{\frac{m+1}{m}}(\Omega)\right]^{d}$ satisfies the following bounds: $\forall \mathbf{v}, \forall \mathbf{w} \in\left[L^{m+1}(\Omega)\right]^{d}$,

$$
\|\mathcal{A}(\boldsymbol{v})\|_{\left[L^{\frac{m+1}{m}}(\Omega)\right]^{d}} \leq \frac{\mu}{\rho}\left\|K^{-1}\right\|\|\boldsymbol{v}\|_{\left[L^{\frac{m+1}{m}}(\Omega)\right]^{d}}+\frac{\beta}{\rho}\|\boldsymbol{v}\|_{\left[L^{m+1}(\Omega)\right]^{d}}^{m}
$$

and

$$
\begin{aligned}
& |\mathcal{A}(\boldsymbol{v})-\mathcal{A}(\boldsymbol{w})| \\
& \quad \leq\left(\frac{\mu}{\rho}\left\|K^{-1}\right\|_{L^{\infty}(\Omega)}+\frac{\beta}{\rho} m 2^{m-2}\left[|\boldsymbol{v}|^{m-1}+|\boldsymbol{w}|^{m-1}\right]\right)|\boldsymbol{v}-\boldsymbol{w}|, \quad \text { a.e. }
\end{aligned}
$$

Proof Let $\boldsymbol{u}, \boldsymbol{v} \in\left[L^{m+1}(\Omega)\right]^{d} . \mathbf{v} \in\left[L^{m+1}(\Omega)\right]^{d} \Longrightarrow \mathcal{A}(\mathbf{v}) \in L^{\frac{m+1}{m}}(\Omega)$. Therefore,

$$
\begin{aligned}
& \mid\left\langle\mathcal{A}(\mathbf{v}),\left.\boldsymbol{u}\right|_{\left[L^{\frac{m+1}{m}}(\Omega)\right]^{d} \times\left[L^{m+1}(\Omega)\right]^{d}}\right| \\
& \quad=\left|\int_{\Omega}\left[\frac{\mu}{\rho} K^{-1} \mathbf{v}+\frac{\beta}{\rho}|\boldsymbol{v}|^{m-1} \mathbf{v}\right] \mathbf{u} d \mathbf{x}\right| \\
& \quad \leq \frac{\mu}{\rho}\left\|K^{-1}\right\|_{L^{\infty}(\Omega)} \int_{\Omega}|\boldsymbol{u} \boldsymbol{v}| d \mathbf{x}+\frac{\beta}{\rho} \int_{\Omega}|\boldsymbol{v}|^{m}|\boldsymbol{u}| d \mathbf{x}
\end{aligned}
$$




$$
\begin{aligned}
\leq & \frac{\mu}{\rho}\left\|K^{-1}\right\|_{L^{\infty}(\Omega)}\|\boldsymbol{u}\|_{\left[L^{m+1}(\Omega)\right]^{d}}\|\boldsymbol{v}\|_{\left[L^{\frac{m+1}{m}}(\Omega)\right]^{d}} \\
& +\frac{\beta}{\rho}\|\boldsymbol{u}\|_{\left[L^{m+1}(\Omega)\right]^{d}}\|\boldsymbol{v}\|_{\left[L^{m+1}(\Omega)\right]^{d}}^{m}
\end{aligned}
$$

Hence,

$$
\begin{aligned}
& \left|\langle\mathcal{A}(\boldsymbol{v}), \boldsymbol{u}\rangle_{\left[L^{\frac{m+1}{m}}(\Omega)\right]^{d} \times\left[L^{m+1}(\Omega)\right]^{d}}\right| \\
& \quad \leq\left[\frac{\mu}{\rho}\left\|K^{-1}\right\|_{L^{\infty}(\Omega)}\|\boldsymbol{v}\|_{\left[L^{\frac{m+1}{m}}(\Omega)\right]^{d}}+\frac{\beta}{\rho}\|\boldsymbol{v}\|_{\left[L^{m+1}(\Omega)\right]^{d}}^{m}\right]\|\boldsymbol{u}\|_{\left[L^{m+1}(\Omega)\right]^{d}} .
\end{aligned}
$$

Using dual norm representation (22) and the last line above, we deduce (40). The estimate in (41) follows from (38).

Lemma 4.2 The map $\boldsymbol{u} \mapsto \mathcal{A}\left(\boldsymbol{u}+\boldsymbol{u}_{\ell}\right)$ defined in (17) is strongly monotone, that is, for all $\boldsymbol{u}, \boldsymbol{v} \in\left[L^{m+1}(\Omega)\right]^{d}$, we have

$$
\int_{\Omega}\left(\mathcal{A}\left(\boldsymbol{u}+\boldsymbol{u}_{\ell}\right)-\mathcal{A}\left(\boldsymbol{v}+\boldsymbol{v}_{\ell}\right)\right) \cdot(\boldsymbol{u}-\boldsymbol{v}) d \mathbf{x} \geq \frac{\mu}{\rho} \lambda_{s}\|\boldsymbol{u}-\boldsymbol{v}\|_{\left[L^{m}(\Omega)\right]^{d}}^{2} .
$$

Proof Define $F:\left[L^{m+1}(\Omega)\right]^{d} \longrightarrow \mathbb{R}$ by

$$
F(\boldsymbol{v})=\frac{\mu}{2 \rho} \int_{\Omega} K^{-1} \boldsymbol{v} \cdot \boldsymbol{v} d \mathbf{x}+\frac{\beta}{\rho(m+1)} \int_{\Omega}|\boldsymbol{v}|^{m+1} d \mathbf{x}, \quad \forall \boldsymbol{v} \in\left[L^{m+1}(\Omega)\right]^{d} .
$$

For all $\boldsymbol{v}, \boldsymbol{w} \in\left[L^{m+1}(\Omega)\right]^{d}$ and $h \in \mathbb{R}$, we have

$$
\begin{aligned}
\frac{F(\boldsymbol{v}+h \boldsymbol{w})-F(\boldsymbol{v})}{h}= & \frac{\mu}{\rho} \int_{\Omega}\left(K^{-1} \boldsymbol{v}\right) \cdot \boldsymbol{w} d \mathbf{x}+\frac{h \mu}{2 \rho} \int_{\Omega} K^{-1} \boldsymbol{w} \cdot \boldsymbol{w} d \mathbf{x} \\
& +\frac{\beta}{\rho(m+1)} \int_{\Omega} \frac{\left(|\boldsymbol{v}+h \boldsymbol{w}|^{m+1}-|\boldsymbol{v}|^{m+1}\right)}{h} d \mathbf{x} .
\end{aligned}
$$

Therefore,

$$
\begin{aligned}
\lim _{h \rightarrow 0} \frac{F(\boldsymbol{v}+h \boldsymbol{w})-F(\boldsymbol{v})}{h}= & \frac{\mu}{\rho} \int_{\Omega} K^{-1} \boldsymbol{v} \cdot \boldsymbol{w} d \mathbf{x} \\
& +\frac{\beta}{\rho(m+1)} \lim _{h \rightarrow 0} \int_{\Omega} \frac{\left(|\boldsymbol{v}+h \boldsymbol{w}|^{m+1}-|\boldsymbol{v}|^{m+1}\right)}{h} d \mathbf{x} .
\end{aligned}
$$

Thanks to the Lebesgue convergence theorem [29], we get

$$
\begin{aligned}
F^{\prime}(\boldsymbol{v}) \cdot \boldsymbol{w} & =\frac{\mu}{\rho} \int_{\Omega} K^{-1} \boldsymbol{v} \cdot \boldsymbol{w} d \mathbf{x}+\frac{\beta}{\rho(m+1)} \int_{\Omega} \lim _{h \rightarrow 0} \frac{\left(|\boldsymbol{v}+h \boldsymbol{w}|^{m+1}-|\boldsymbol{v}|^{m+1}\right)}{h} d \mathbf{x} \\
& =\frac{\mu}{\rho} \int_{\Omega} K^{-1} \boldsymbol{v} \cdot \boldsymbol{w} d \mathbf{x}+\left.\frac{\beta}{\rho(m+1)} \int_{\Omega} \frac{d}{d h}|\boldsymbol{v}+h \boldsymbol{w}|^{m+1}\right|_{h=0} \\
& =\frac{\mu}{\rho} \int_{\Omega} K^{-1} \boldsymbol{v} \cdot \boldsymbol{w} d \mathbf{x}+\left.\frac{\beta}{\rho(m+1)} \int_{\Omega} \frac{d}{d h}[(\boldsymbol{v}+h \boldsymbol{w}) \cdot(\boldsymbol{v}+h \boldsymbol{w})]^{\frac{m+1}{2}}\right|_{h=0} \\
& =\frac{\mu}{\rho} \int_{\Omega} K^{-1} \boldsymbol{v} \cdot \boldsymbol{w} d \mathbf{x}+\frac{\beta}{\rho(m+1)} \int_{\Omega} \frac{m+1}{2}|\boldsymbol{v}|^{m-1}(\boldsymbol{v} \cdot \boldsymbol{w}+\boldsymbol{v} \cdot \boldsymbol{w}) d \mathbf{x} .
\end{aligned}
$$


Hence,

$$
F^{\prime}(\boldsymbol{v}) \cdot \boldsymbol{w}=\frac{\mu}{\rho} \int_{\Omega} K^{-1} \boldsymbol{v} \cdot \boldsymbol{w} d \mathbf{x}+\frac{\beta}{\rho} \int_{\Omega}|\boldsymbol{v}|^{m-1}(\boldsymbol{v} \cdot \boldsymbol{w}) d \mathbf{x} .
$$

We have

$$
\begin{aligned}
F^{\prime \prime}(\boldsymbol{v}) \cdot(\mathbf{z}, \boldsymbol{w})= & \lim _{h \rightarrow 0} \frac{f^{\prime}(\boldsymbol{v}+h \mathbf{z}) \boldsymbol{w}-f^{\prime}(\boldsymbol{v}) \boldsymbol{w}}{h} \\
= & \frac{\mu}{\rho} \int_{\Omega} K^{-1} \boldsymbol{z} \cdot \boldsymbol{w} d \mathbf{x}+\frac{\beta}{\rho} \int_{\Omega}|\boldsymbol{v}|^{m-1}(\mathbf{z} \cdot \boldsymbol{w}) d \mathbf{x} \\
& +\frac{\beta}{\rho} \int_{\Omega} \lim _{h \rightarrow 0}\left[\frac{|\boldsymbol{v}+h \mathbf{z}|^{m-1}(\boldsymbol{v} \cdot \boldsymbol{w})-|\boldsymbol{v}|^{m-1}}{h}\right](\boldsymbol{v} \cdot \boldsymbol{w}) d \mathbf{x} \\
= & \frac{\mu}{\rho} \int_{\Omega} K^{-1} \boldsymbol{z} \cdot \boldsymbol{w} d \mathbf{x}+\frac{\beta}{\rho} \int_{\Omega}|\boldsymbol{v}|^{m-1}(\mathbf{z} \cdot \boldsymbol{w}) d \mathbf{x} \\
& +\left.\int_{\Omega} \frac{d}{d h}|\boldsymbol{v}+h \mathbf{z}|^{m-1}\right|_{h=0}(\boldsymbol{v} \cdot \boldsymbol{w}) d \mathbf{x} \\
= & \frac{\mu}{\rho} \int_{\Omega} K^{-1} \mathbf{z} \cdot \boldsymbol{w} d \mathbf{x}+\frac{\beta}{\rho} \int_{\Omega}|\boldsymbol{v}|^{m-1}(\mathbf{z} \cdot \boldsymbol{w}) d \mathbf{x} \\
& +\left.\int_{\Omega} \frac{d}{d h}[(\boldsymbol{v}+h \mathbf{z}) \cdot(\boldsymbol{v}+h \mathbf{z})]^{\frac{m-1}{2}}\right|_{h=0}(\boldsymbol{v} \cdot \boldsymbol{w}) d \mathbf{x} .
\end{aligned}
$$

Consequently,

$$
\begin{aligned}
F^{\prime \prime}(\mathbf{v}) \cdot(\mathbf{z}, \boldsymbol{w})= & \frac{\mu}{\rho} \int_{\Omega} K^{-1} \mathbf{z} \cdot \boldsymbol{w} d \mathbf{x}+\frac{\beta}{\rho} \int_{\Omega}|\boldsymbol{v}|^{m-1}(\mathbf{z} \cdot \boldsymbol{w}) d \mathbf{x} \\
& +\left.\frac{\beta}{\rho} \int_{\Omega} \frac{m-1}{2}|\boldsymbol{v}+h \mathbf{z}|^{m-3}[(\mathbf{v}+h \mathbf{z}) \cdot \mathbf{z}+(\boldsymbol{v}+h \mathbf{z}) \cdot \boldsymbol{z}]\right|_{h=0}(\mathbf{v} \cdot \boldsymbol{w}) d \mathbf{x} .
\end{aligned}
$$

It follows that

$$
\begin{aligned}
F^{\prime \prime}(\boldsymbol{v}) \cdot(\mathbf{z}, \boldsymbol{w})= & \frac{\mu}{\rho} \int_{\Omega} K^{-1} \boldsymbol{z} \cdot \boldsymbol{w} d \mathbf{x}+\frac{\beta}{\rho} \int_{\Omega}|\boldsymbol{v}|^{m-1}(\mathbf{z} \cdot \boldsymbol{w}) d \mathbf{x} \\
& +\frac{\beta}{\rho}(m-1) \int_{\Omega}|\boldsymbol{v}|^{m-3}(\boldsymbol{v} \cdot \mathbf{z})(\boldsymbol{v} \cdot \boldsymbol{w}) d \mathbf{x} .
\end{aligned}
$$

Therefore,

$$
F^{\prime \prime}(0) \cdot(\boldsymbol{w}, \boldsymbol{z})=\frac{\mu}{\rho} \int_{\Omega} K^{-1} \boldsymbol{z} \cdot \boldsymbol{w} d \mathbf{x}, \quad \forall \boldsymbol{w}, \forall \mathbf{z} \in\left[L^{m+1}(\Omega)\right]^{d}
$$

Now we observe that $F^{\prime \prime}$ is positive definite and symmetric. Indeed,

$$
F^{\prime \prime}(\boldsymbol{v}) \cdot(\boldsymbol{w}, \boldsymbol{w}) \geq \frac{\mu}{\rho} \lambda_{s}\|\boldsymbol{w}\|_{L^{2}}^{2}, \quad \forall \boldsymbol{v}, \boldsymbol{w} \in\left[L^{m+1}(\Omega)\right]^{d}
$$

Let $\boldsymbol{u}, \boldsymbol{v}$ be in $\left[L^{m+1}(\Omega)\right]^{d}$. Set $\hat{\boldsymbol{u}}=\boldsymbol{u}+\boldsymbol{u}_{\ell}$ and $\hat{\boldsymbol{v}}=\boldsymbol{v}+\boldsymbol{u}_{\ell}$, where $\boldsymbol{u}_{\ell}$ is fixed in $\left[L^{m+1}(\Omega)\right]^{d}$. Then

$$
\left(F^{\prime}(\hat{\boldsymbol{u}})-F^{\prime}(\hat{\boldsymbol{v}})\right) \cdot(\hat{\boldsymbol{u}}-\hat{\boldsymbol{v}})
$$




$$
\begin{aligned}
= & F^{\prime}(\hat{\boldsymbol{u}}) \cdot(\hat{\boldsymbol{u}}-\hat{\boldsymbol{v}})-F^{\prime}(\hat{\boldsymbol{v}}) \cdot(\hat{\boldsymbol{u}}-\hat{\boldsymbol{v}}) \\
= & \frac{\mu}{\rho} \int_{\Omega}\left(K^{-1} \hat{\boldsymbol{u}}\right) \cdot(\hat{\boldsymbol{u}}-\hat{\mathbf{v}}) d \mathbf{x}+\frac{\beta}{\rho} \int_{\Omega}|\hat{\boldsymbol{u}}|^{m-1}(\hat{\boldsymbol{u}} \cdot(\hat{\boldsymbol{u}}-\hat{\mathbf{v}})) d \mathbf{x} \\
& -\left[\frac{\mu}{\rho} \int_{\Omega}\left(K^{-1} \hat{\mathbf{v}}\right) \cdot(\hat{\boldsymbol{u}}-\hat{\mathbf{v}}) d \mathbf{x}+\frac{\beta}{\rho} \int_{\Omega}|\hat{\mathbf{v}}|^{m-1}(\hat{\boldsymbol{v}} \cdot(\hat{\boldsymbol{u}}-\hat{\mathbf{v}})) d \mathbf{x}\right] \\
= & \frac{\mu}{\rho} \int_{\Omega}\left(K^{-1}(\hat{\boldsymbol{u}}-\hat{\mathbf{v}}) \cdot(\hat{\boldsymbol{u}}-\hat{\mathbf{v}}) d \mathbf{x}\right. \\
& +\frac{\beta}{\rho} \int_{\Omega}\left(|\hat{\boldsymbol{u}}|^{m-1} \hat{\boldsymbol{u}}-|\hat{\mathbf{v}}|^{m-1} \hat{\boldsymbol{v}}\right) \cdot(\hat{\boldsymbol{u}}-\hat{\mathbf{v}}) d \mathbf{x} .
\end{aligned}
$$

That is,

$$
\left(F^{\prime}(\hat{\boldsymbol{u}})-F^{\prime}(\hat{\boldsymbol{v}})\right) \cdot(\hat{\boldsymbol{u}}-\hat{\boldsymbol{v}})=\int_{\Omega}(\mathcal{A}(\hat{\boldsymbol{u}})-\mathcal{A}(\hat{\boldsymbol{v}})) \cdot(\boldsymbol{u}-\boldsymbol{v}) d \mathbf{x} .
$$

Furthermore, by the mean value theorem, we have

$$
\left(F^{\prime}(\hat{\boldsymbol{u}})-F^{\prime}(\hat{\boldsymbol{v}})\right) \cdot(\hat{\mathbf{u}}-\hat{\mathbf{v}})=\int_{0}^{1} F^{\prime \prime}(\hat{\boldsymbol{v}}+\alpha(\hat{\mathbf{u}}-\hat{\mathbf{v}})) \cdot(\hat{\boldsymbol{u}}-\hat{\mathbf{v}}, \hat{\mathbf{u}}-\hat{\mathbf{v}}) d \alpha .
$$

Therefore, (47) becomes

$$
\int_{\Omega}(\mathcal{A}(\hat{\boldsymbol{u}})-\mathcal{A}(\hat{\boldsymbol{v}})) \cdot(\boldsymbol{u}-\boldsymbol{v}) d \mathbf{x}=\int_{0}^{1} F^{\prime \prime}(\hat{\boldsymbol{v}}+\alpha(\hat{\boldsymbol{u}}-\hat{\boldsymbol{v}})) \cdot(\hat{\boldsymbol{u}}-\hat{\mathbf{v}}, \hat{\mathbf{u}}-\hat{\boldsymbol{v}}) d \alpha .
$$

Thanks to (46), equation (49) becomes

$$
\int_{\Omega}(\mathcal{A}(\hat{\boldsymbol{u}})-\mathcal{A}(\hat{\boldsymbol{v}})) \cdot(\boldsymbol{u}-\boldsymbol{v}) d \mathbf{x} \geq \int_{0}^{1} \frac{\mu}{\rho} \lambda_{s}\|\hat{\boldsymbol{u}}-\hat{\boldsymbol{v}}\|_{L^{2}}^{2} d \alpha \geq \frac{\mu}{\rho} \lambda_{s}\|\hat{\boldsymbol{u}}-\hat{\boldsymbol{v}}\|_{\left[L^{m}(\Omega)\right] d^{2}}^{2}
$$

Hence, we have (42) since $\hat{\boldsymbol{u}}-\hat{\boldsymbol{v}}=\boldsymbol{u}-\boldsymbol{v}$.

Lemma 4.3 The map $\boldsymbol{u} \mapsto \mathcal{A}\left(\boldsymbol{u}+\boldsymbol{u}_{\ell}\right)$ in (17) is coercive in $\left[L^{m+1}(\Omega)\right]^{d}$, for any $\boldsymbol{u}_{\ell}$ fixed in $\left[L^{m+1}(\Omega)\right]^{d}$,

$$
\lim _{\|\boldsymbol{u}\|_{\left[L^{m+1}(\Omega)\right]^{d} \rightarrow \infty}}\left(\frac{1}{\|\boldsymbol{u}\|_{\left[L^{m+1}(\Omega)\right]^{d}}} \int_{\Omega} \mathcal{A}\left(\boldsymbol{u}+\boldsymbol{u}_{\ell}\right) \cdot \boldsymbol{u} d \mathbf{x}\right)=\infty .
$$

Proof Let $\boldsymbol{u} \in\left[L^{m+1}(\Omega)\right]^{d}$ be arbitrary chosen and assign $\hat{\boldsymbol{u}}=\boldsymbol{u}+\boldsymbol{u}_{\ell}$. Then

$$
\int_{\Omega} \mathcal{A}(\hat{\mathbf{u}}) \cdot \mathbf{u} d \mathbf{x}=\int_{\Omega} \mathcal{A}(\hat{\mathbf{u}}) \hat{\mathbf{u}} d \mathbf{x}-\int_{\Omega} \mathcal{A}(\hat{\mathbf{u}}) \mathbf{u}_{\ell} d \mathbf{x}
$$

Thanks to (43), we see that

$$
\begin{aligned}
F^{\prime}(\hat{\boldsymbol{u}}) \cdot \hat{\boldsymbol{u}} & =\frac{\mu}{\rho} \int_{\Omega} K^{-1} \hat{\boldsymbol{u}} \cdot \hat{\mathbf{u}} d \mathbf{x}+\frac{\beta}{\rho} \int_{\Omega}|\hat{\boldsymbol{u}}|^{m-1}(\hat{\boldsymbol{u}} \cdot \hat{\boldsymbol{u}}) d \mathbf{x} \\
& =\int_{\Omega}\left(\frac{\mu}{\rho} K^{-1} \hat{\boldsymbol{u}}+\frac{\beta}{\rho}|\boldsymbol{u}|^{m-1} \hat{\boldsymbol{u}}\right) \cdot \hat{\mathbf{u}} d \mathbf{x}
\end{aligned}
$$




$$
=\int_{\Omega} \mathcal{A}(\hat{\boldsymbol{u}}) \cdot \hat{\mathbf{u}} d \mathbf{x}
$$

With the last line above, (52) becomes

$$
\int_{\Omega} \mathcal{A}(\hat{\boldsymbol{u}}) \cdot \boldsymbol{u} d \mathbf{x}=F^{\prime}(\hat{\boldsymbol{u}}) \cdot \hat{\boldsymbol{u}}-\int_{\Omega} \mathcal{A}(\hat{\boldsymbol{u}}) \mathbf{u}_{\ell} d \mathbf{x} .
$$

We have

$$
\begin{aligned}
F^{\prime}(\hat{\boldsymbol{u}}) \cdot \hat{\boldsymbol{u}} & =\frac{\mu}{\rho} \int_{\Omega}\left(K^{-1} \hat{\boldsymbol{u}}\right) \cdot \hat{\mathbf{u}} d \mathbf{x}+\frac{\beta}{\rho} \int_{\Omega}|\hat{\boldsymbol{u}}|^{m-1}(\hat{\boldsymbol{u}} \cdot \hat{\boldsymbol{u}}) d \mathbf{x} \\
& =\frac{\mu}{\rho} \int_{\Omega}\left(K^{-1} \hat{\boldsymbol{u}}\right) \cdot \hat{\boldsymbol{u}} d \mathbf{x}+\frac{\beta}{\rho} \int_{\Omega}|\hat{\boldsymbol{u}}|^{m+1} d \mathbf{x} .
\end{aligned}
$$

This implies

$$
\begin{aligned}
F^{\prime}(\hat{\boldsymbol{u}}) \cdot \hat{\boldsymbol{u}} & \geq \frac{\mu}{\rho} \lambda_{s}\|\hat{\boldsymbol{u}}\|_{L^{2}}^{2}+\frac{\beta}{\rho}\|\hat{\boldsymbol{u}}\|_{\left[L^{m+1}(\Omega)\right]^{d}}^{m+1} \\
& \geq \frac{C \mu}{\rho} \lambda_{s}\|\hat{\boldsymbol{u}}\|_{\left[L^{m}(\Omega)\right]^{d}}^{2}+\frac{\beta}{\rho}\|\hat{\boldsymbol{u}}\|_{\left[L^{m+1}(\Omega)\right]^{d}}^{m+1}, \quad m \leq 2 .
\end{aligned}
$$

Now, we estimate the second term on the right-hand side of (53).

$$
\begin{aligned}
\int_{\Omega} \mathcal{A}(\hat{\boldsymbol{u}}) \boldsymbol{u}_{\ell} d \mathbf{x} \leq & \|\mathcal{A}(\hat{\boldsymbol{u}})\|_{\left[L^{\left.\frac{m+1}{m}(\Omega)\right]^{d}}\right.}\left\|\boldsymbol{u}_{\ell}\right\|_{\left[L^{m+1}(\Omega)\right]^{d}} \\
\leq & \left(\frac{\mu}{\rho}\left\|K^{-1}\right\|_{L^{\infty}(\Omega)}\|\hat{\boldsymbol{u}}\|_{\left[L^{\frac{m+1}{m}}(\Omega)\right]^{d}}+\frac{\beta}{\rho}\|\hat{\boldsymbol{u}}\|_{\left[L^{m+1}(\Omega)\right]^{d}}^{m}\left\|\boldsymbol{u}_{\ell}\right\|_{\left[L^{m+1}(\Omega)\right]^{d}}\right. \\
\leq & \frac{\mu}{\rho}\left\|K^{-1}\right\|_{L^{\infty}(\Omega)}\|\hat{\boldsymbol{u}}\|_{\left[L^{\left.\frac{m+1}{m}(\Omega)\right]^{d}}\right.}\left\|\boldsymbol{u}_{\ell}\right\|_{\left[L^{m+1}(\Omega)\right]^{d}} \\
& +\frac{\beta}{\rho}\|\hat{\boldsymbol{u}}\|_{\left[L^{m+1}(\Omega)\right]^{d}}^{m}\left\|\boldsymbol{u}_{\ell}\right\|_{\left[L^{m+1}(\Omega)\right]^{d}}
\end{aligned}
$$

Substituting (54) and (55) into (53), we get

$$
\begin{aligned}
\int_{\Omega} \mathcal{A}(\hat{\boldsymbol{u}}) \cdot \boldsymbol{u} d \mathbf{x} \geq & \frac{C \mu}{\rho} \lambda_{s}\|\hat{\boldsymbol{u}}\|_{\left[L^{m}(\Omega)\right]^{d}}^{2}+\frac{\beta}{\rho}\|\hat{\boldsymbol{u}}\|_{\left[L^{m+1}(\Omega)\right]^{d}}^{m+1} \\
& -\frac{\mu}{\rho}\left\|K^{-1}\right\|_{L^{\infty}(\Omega)}\|\hat{\boldsymbol{u}}\|_{\left[L^{\frac{m+1}{m}}(\Omega)\right]^{d}}\left\|\boldsymbol{u}_{\ell}\right\|_{\left[L^{m+1}(\Omega)\right]^{d}} \\
& -\frac{\beta}{\rho}\|\hat{\boldsymbol{u}}\|_{\left[L^{m+1}(\Omega)\right]^{m}}^{m}\left\|\boldsymbol{u}_{\ell}\right\|_{\left[L^{m+1}(\Omega)\right]^{d}}
\end{aligned}
$$

Hence,

$$
\begin{aligned}
\int_{\Omega} \mathcal{A}(\hat{\boldsymbol{u}}) \cdot \boldsymbol{u} d \mathbf{x} \geq & \frac{\beta}{\rho}\|\hat{\boldsymbol{u}}\|_{\left[L^{m+1}(\Omega)\right]^{d}}^{m}\left(\|\hat{\boldsymbol{u}}\|_{\left[L^{m+1}(\Omega)\right]^{d}}-\left\|\boldsymbol{u}_{\ell}\right\|_{\left[L^{m+1}(\Omega)\right]^{d}}\right) \\
& +\frac{\mu}{\rho} C \lambda_{s}\|\hat{\boldsymbol{u}}\|_{\left[L^{m}(\Omega)\right]^{d}}\left(\|\hat{\boldsymbol{u}}\|_{\left[L^{m}(\Omega)\right]^{d}}-\frac{\left\|K^{-1}\right\|_{L^{\infty}(\Omega)}\left\|\boldsymbol{u}_{\ell}\right\|_{\left[L^{m+1}(\Omega)\right]^{d}}}{C \lambda_{s}}\right) .
\end{aligned}
$$

If $\|\boldsymbol{u}\|_{\left[L^{m}(\Omega)\right]^{d}} \longrightarrow \infty$, then

$$
\frac{\mu}{\rho} C \lambda_{s}\|\hat{\boldsymbol{u}}\|_{\left[L^{m}(\Omega)\right]^{d}}\left(\|\hat{\boldsymbol{u}}\|_{\left[L^{m}(\Omega)\right]^{d}}-\frac{\left\|K^{-1}\right\|_{L^{\infty}(\Omega)}\left\|\boldsymbol{u}_{\ell}\right\|_{\left[L^{m+1}(\Omega)\right]^{d}}}{C \lambda_{s}}\right) \geq 0 .
$$


Therefore,

$$
\int_{\Omega} \mathcal{A}(\hat{\boldsymbol{u}}) \boldsymbol{u} d \mathbf{x} \geq \frac{\beta}{\rho}\|\hat{\boldsymbol{u}}\|_{\left[L^{m+1}(\Omega)\right]^{d}}\|\hat{\boldsymbol{u}}\|_{\left[L^{m+1}(\Omega)\right]^{d}}^{m-1}\left(\|\hat{\boldsymbol{u}}\|_{\left[L^{m+1}(\Omega)\right]^{d}}-\left\|\boldsymbol{u}_{\ell}\right\|_{\left[L^{m+1}(\Omega)\right]^{d}}\right) .
$$

Recalling that

$$
\hat{\boldsymbol{u}}=\boldsymbol{u}+\boldsymbol{u}_{\ell} \Longrightarrow\|\hat{\boldsymbol{u}}\|_{\left[L^{m+1}(\Omega)\right]^{d}} \geq\|\boldsymbol{u}\|_{\left[L^{m+1}(\Omega)\right]^{d}}-\left\|\boldsymbol{u}_{\ell}\right\|_{\left[L^{m+1}(\Omega)\right]^{d}} .
$$

As $\|\boldsymbol{u}\|_{\left[L^{m}(\Omega)\right]^{d}} \longrightarrow \infty$, we obtain

$$
\|\boldsymbol{u}\|_{\left[L^{m+1}(\Omega)\right]^{d}}-\left\|\boldsymbol{u}_{\ell}\right\|_{\left[L^{m+1}(\Omega)\right]^{d}} \geq \frac{1}{2}\|\boldsymbol{u}\|_{\left[L^{m+1}(\Omega)\right]^{d}}
$$

since $\|\boldsymbol{u}\|_{\left[L^{m+1}(\Omega)\right]^{d}} \geq 2\left\|\boldsymbol{u}_{\ell}\right\|_{\left[L^{m+1}(\Omega)\right]^{d}}$.

Hence,

$$
\int_{\Omega} \mathcal{A}(\hat{\boldsymbol{u}}) \boldsymbol{u} d \mathbf{x} \geq \frac{\beta}{2 \rho}\|\boldsymbol{u}\|_{\left[L^{m+1}(\Omega)\right]^{d}}\|\hat{\boldsymbol{u}}\|_{\left[L^{m+1}(\Omega)\right]^{d}}^{m-1}\left(\|\hat{\boldsymbol{u}}\|_{\left[L^{m+1}(\Omega)\right]^{d}}-\left\|\boldsymbol{u}_{\ell}\right\|_{\left[L^{m+1}(\Omega)\right]^{d}}\right) .
$$

It follows that

$$
\frac{1}{\|\boldsymbol{u}\|_{\left[L^{m+1}(\Omega)\right]^{d}}} \int_{\Omega} \mathcal{A}(\hat{\boldsymbol{u}}) \boldsymbol{u} d \boldsymbol{x} \geq\|\hat{\boldsymbol{u}}\|_{\left[L^{m+1}(\Omega)\right]^{d}}^{m-1}\left(\|\hat{\boldsymbol{u}}\|_{\left[L^{m+1}(\Omega)\right]^{d}}-\left\|\boldsymbol{u}_{\ell}\right\|_{\left[L^{m+1}(\Omega)\right]^{d}}\right) .
$$

Letting $\|\boldsymbol{u}\|_{\left[L^{m+1}(\Omega)\right]^{d}} \longrightarrow \infty$, the desired result (51) is achieved.

Proposition 4.1 For $\mathbf{u}_{\ell}$ fixed in $\left[L^{m+1}(\Omega)\right]^{d}$, the map

$$
\theta \mapsto \int_{\Omega} \mathcal{A}\left(\boldsymbol{u}+\boldsymbol{u}_{\ell}+\theta \boldsymbol{v}\right) \cdot \boldsymbol{w} d \mathbf{x},
$$

is continuous on $\mathbb{R}$ for all $\mathbf{u}, \boldsymbol{v}, \boldsymbol{w} \in\left[L^{m+1}(\Omega)\right]^{d}$. In other words, $\mathcal{A}$ is hemi-continuous in $\left[L^{m+1}(\Omega)\right]^{d}$.

Proof Let $\boldsymbol{u}$ and $\boldsymbol{v}$ in $\left[L^{m+1}(\Omega)\right]^{d}$ be arbitrary functions and assign $\hat{\boldsymbol{u}}=\boldsymbol{u}+\boldsymbol{u}_{\ell}$.

For any $\theta_{1}, \theta_{2} \in \mathbb{R}$,

$$
\begin{aligned}
\int_{\Omega} \mathcal{A}\left(\hat{\boldsymbol{u}}+\theta_{1} \mathbf{v}\right) \cdot \boldsymbol{v} d \mathbf{x} & =\int_{\Omega}\left(\frac{\mu}{\rho} K^{-1}\left(\hat{\boldsymbol{u}}+\theta_{1} \mathbf{v}\right)+\frac{\beta}{\rho}\left|\hat{\boldsymbol{u}}+\theta_{1} \mathbf{v}\right|^{m-1} \cdot\left(\hat{\boldsymbol{u}}+\theta_{1} \mathbf{v}\right)\right) \cdot \boldsymbol{v} d \mathbf{x} \\
& =\frac{\mu}{\rho} \int_{\Omega} K^{-1}\left(\hat{\boldsymbol{u}}+\theta_{1} \mathbf{v}\right) \cdot \mathbf{v} d \mathbf{x}+\frac{\beta}{\rho} \int_{\Omega}\left|\hat{\boldsymbol{u}}+\theta_{1} \mathbf{v}\right|^{m-1}\left(\hat{\boldsymbol{u}}+\theta_{1} \mathbf{v}\right) \cdot \mathbf{v} d \mathbf{x} .
\end{aligned}
$$

Thanks to (43), the last line becomes

$$
\int_{\Omega} \mathcal{A}\left(\hat{\boldsymbol{u}}+\theta_{1} \mathbf{v}\right) \cdot \mathbf{v} d \mathbf{x}=F^{\prime}\left(\hat{\boldsymbol{u}}+\theta_{1} \mathbf{v}\right) \cdot \mathbf{v} .
$$

Similarly,

$$
\int_{\Omega} \mathcal{A}\left(\hat{\boldsymbol{u}}+\theta_{2} \mathbf{v}\right) \cdot \mathbf{v} d \mathbf{x}=F^{\prime}\left(\hat{\boldsymbol{u}}+\theta_{2} \mathbf{v}\right) \cdot \mathbf{v} .
$$


Therefore,

$$
\int_{\Omega}\left(\mathcal{A}\left(\hat{\boldsymbol{u}}+\theta_{1} \boldsymbol{v}\right)-\mathcal{A}\left(\hat{\boldsymbol{u}}+\theta_{2} \boldsymbol{v}\right)\right) \cdot \boldsymbol{v} d \mathbf{x}=\left(F^{\prime}\left(\hat{\boldsymbol{u}}+\theta_{1} \boldsymbol{v}\right)-F^{\prime}\left(\hat{\boldsymbol{u}}+\theta_{2} \boldsymbol{v}\right)\right) \cdot \boldsymbol{v} .
$$

Now, we define

$$
H(\theta)=F^{\prime}\left(\theta\left(\hat{\boldsymbol{u}}+\theta_{1} \mathbf{v}\right)+(1-\theta)\left(\hat{\boldsymbol{u}}+\theta_{2} \boldsymbol{v}\right)\right), \quad \theta \in[0,1] .
$$

By the mean value theorem [29],

$$
H(1)-H(0)=H^{\prime}(\alpha), \quad \alpha \in(0,1)
$$

In fact,

$$
\begin{aligned}
H^{\prime}(\alpha) & =F^{\prime \prime}\left(\alpha\left(\hat{\boldsymbol{u}}+\theta_{1} \mathbf{v}\right)+(1-\alpha)\left(\hat{\boldsymbol{u}}+\theta_{2} \boldsymbol{v}\right)\right) \cdot\left(\theta_{1}-\theta_{2}\right) \mathbf{v} \\
& =F^{\prime \prime}\left(\hat{\boldsymbol{u}}+\theta_{2} \boldsymbol{v}-\alpha\left(\theta_{2}-\theta_{1}\right) \mathbf{v}\right) \cdot\left(\theta_{1}-\theta_{2}\right) \mathbf{v} .
\end{aligned}
$$

Therefore, (59) becomes

$$
\left(F^{\prime}\left(\hat{\boldsymbol{u}}+\theta_{1} \boldsymbol{v}\right)-F^{\prime}\left(\hat{\boldsymbol{u}}+\theta_{2} \boldsymbol{v}\right)\right) \cdot \mathbf{v}=F^{\prime \prime}\left(\hat{\boldsymbol{u}}+\theta_{2} \boldsymbol{v}-\alpha\left(\theta_{2}-\theta_{1}\right) \mathbf{v}\right) \cdot\left(\theta_{1}-\theta_{2}\right) \mathbf{v}, \quad \alpha \in(0,1)
$$

Using (61), it follows from (58) that

$$
\begin{aligned}
& \int_{\Omega}\left(\mathcal{A}\left(\hat{\boldsymbol{u}}+\theta_{1} \mathbf{v}\right)-\mathcal{A}\left(\hat{\boldsymbol{u}}+\theta_{2} \boldsymbol{v}\right)\right) \cdot \mathbf{v} d \mathbf{x} \\
& \quad=-\left(\theta_{2}-\theta_{1}\right) \int_{0}^{1} F^{\prime \prime}\left(\hat{\boldsymbol{u}}+\theta_{2} \boldsymbol{v}-\alpha\left(\theta_{2}-\theta_{1}\right) \mathbf{v}\right) \cdot(\mathbf{v}, \mathbf{v}) d \alpha .
\end{aligned}
$$

In view of (44), the term on the RHS of (62) goes to zero as $\theta_{2}-\theta_{1}$ goes to zero. Thus Proposition (4.1) is proved.

\subsection{Proof of the main result (Theorem 1.1)}

In view of Lemmas 4.1, 4.2, 4.3 and Proposition 4.1, the existence and uniqueness of $\boldsymbol{u}$ follows from the Minty-Browder theorem [23]. The inf-sup condition in Lemma 3.1 guarantees the existence and uniqueness of $p[21,26]$, so that the pair $(\boldsymbol{u}, p)$ solves Problem (12)-(13).

\section{Stability}

In this section, we prove the stability of the solution in Theorem 1.1.

Theorem 5.1 For any lifting $\boldsymbol{u}_{\ell}$ in $\left[L^{m+1}(\Omega)\right]^{d}$ satisfying (26), the pair of functions $(\boldsymbol{u}, p)$ in Theorem 1.1 satisfies the following estimates:

$$
\begin{aligned}
\|\boldsymbol{u}\|_{\left[L^{m+1}(\Omega)\right]^{d}} \leq & \left(\left\|\boldsymbol{u}_{\ell}\right\|_{\left[L^{m+1}(\Omega)\right]^{d}}^{m+1}+\frac{C(m+1)}{4} \frac{\mu}{\beta} \frac{\left\|K^{-1}\right\|_{L^{\infty}(\Omega)}}{\lambda_{s}}\left\|\boldsymbol{u}_{\ell}\right\|_{\left[L^{m+1}(\Omega)\right]^{d}}^{2}\right. \\
& \left.+\frac{\rho(m+1)}{\beta}\|\boldsymbol{f}\|_{\left[L^{\frac{m+1}{m}}(\Omega)\right]^{d}}\left\|\boldsymbol{u}_{0}\right\|_{\left[L^{m+1}(\Omega)\right]^{d}}\right)^{\frac{1}{m+1}}
\end{aligned}
$$


where $\mathbf{u}_{\ell}$ satisfies the bound in (26) and

$$
\|\nabla p\|_{\left[L^{\left.\frac{m+1}{m}(\Omega)\right]^{d}}\right.} \leq \frac{\mu}{\rho}\left\|K^{-}\right\|_{L^{\infty}}\|\boldsymbol{u}\|_{\left[L^{\frac{m+1}{m}}(\Omega)\right]^{d}}+\frac{\beta}{\rho}\|\boldsymbol{u}\|_{\left[L^{m+1}(\Omega)\right]^{d}}^{2}+\|\boldsymbol{f}\|_{\left[L^{\frac{m+1}{m}}(\Omega)\right]^{d^{2}}} .
$$

Proof Setting $\boldsymbol{\varphi}=\boldsymbol{u}_{0}$ in (33) gives

$$
\int_{\Omega} \mathcal{A}\left(\boldsymbol{u}_{0}+\boldsymbol{u}_{\ell}\right) \cdot \boldsymbol{u}_{0} d \mathbf{x}=\int_{\Omega} \boldsymbol{f} \cdot \boldsymbol{u}_{0} d \mathbf{x}
$$

Therefore,

$$
\begin{aligned}
\int_{\Omega} \mathcal{A}(\boldsymbol{u}) \cdot \mathbf{u} d \mathbf{x} & =\int_{\Omega} \mathcal{A}\left(\boldsymbol{u}_{0}+\mathbf{u}_{\ell}\right) \cdot \mathbf{u}_{0} d \mathbf{x}+\int_{\Omega} \mathcal{A}\left(\boldsymbol{u}_{0}+\mathbf{u}_{\ell}\right) \cdot \mathbf{u}_{\ell} d \mathbf{x} \\
& =\int_{\Omega} \boldsymbol{f} \cdot \mathbf{u}_{0} d \mathbf{x}+\int_{\Omega} \mathcal{A}\left(\boldsymbol{u}_{0}+\mathbf{u}_{\ell}\right) \cdot \mathbf{u}_{\ell} d \mathbf{x} .
\end{aligned}
$$

We estimate the LHS of (66) as follows:

$$
\begin{aligned}
\int_{\Omega} \mathcal{A}(\boldsymbol{u}) \cdot \boldsymbol{u} d \mathbf{x} & =\int_{\Omega} \frac{\mu}{\rho} K^{-1} \boldsymbol{u} \cdot \boldsymbol{u} d \mathbf{x}+\int_{\Omega} \frac{\beta}{\rho}|\boldsymbol{u}|^{m-1} \boldsymbol{u} \cdot \boldsymbol{u} d \mathbf{x} \\
& \geq \frac{\mu \lambda_{s}}{\rho}\|\boldsymbol{u}\|_{\left[L^{2}(\Omega)\right]^{d}}^{2}+\frac{\beta}{\rho}\|\boldsymbol{u}\|_{\left[L^{m+1}(\Omega)\right]^{d}}^{m+1} .
\end{aligned}
$$

The RHS of (66) gives the following bound:

$$
\begin{aligned}
\int_{\Omega} \boldsymbol{f} \cdot \boldsymbol{u}_{0} d \boldsymbol{x}+\int_{\Omega} \mathcal{A}(\boldsymbol{u}) \cdot \boldsymbol{u}_{\ell} d \boldsymbol{x} \leq & \|\boldsymbol{f}\|_{\left[L^{\frac{m+1}{m}}(\Omega)\right]^{d}}\left\|\boldsymbol{u}_{0}\right\|_{\left[L^{m+1}(\Omega)\right]^{d}} \\
& +\|\mathcal{A}(\boldsymbol{u})\|_{\left[L^{\frac{m+1}{m}}(\Omega)\right]^{d}}\left\|\boldsymbol{u}_{\ell}\right\|_{\left[L^{m+1}(\Omega)\right]^{d}}
\end{aligned}
$$

In view of (40), we get

$$
\begin{aligned}
\int_{\Omega} \boldsymbol{f} \cdot \boldsymbol{u}_{0} d \boldsymbol{x}+\int_{\Omega} \mathcal{A}(\boldsymbol{u}) \cdot \boldsymbol{u}_{\ell} d \mathbf{x} \leq & \|\boldsymbol{f}\|_{\left[\frac{\left.\frac{m+1}{m}(\Omega)\right]^{d}}{\rho}\left\|\boldsymbol{u}_{0}\right\|_{\left[L^{m+1}(\Omega)\right]^{d}}\right.} \\
& +\frac{\mu}{\rho}\left\|K^{-1}\right\|_{L^{\infty}(\Omega)}\|\boldsymbol{u}\|_{\left[L^{\frac{m+1}{m}}(\Omega)\right]^{d}}\left\|\boldsymbol{u}_{\ell}\right\|_{\left[L^{m+1}(\Omega)\right]^{d}} \\
& +\frac{\beta}{\rho}\|\boldsymbol{u}\|_{\left.\left[L^{m+1}(\Omega)\right]\right]^{m}}^{m}\left\|\boldsymbol{u}_{\ell}\right\|_{\left[L^{m+1}(\Omega)\right]^{d}}
\end{aligned}
$$

Substituting (67) and (68) into (66) yields

$$
\begin{aligned}
\frac{\mu}{\rho} \lambda_{s}\|\boldsymbol{u}\|_{L^{2}}^{2}+\frac{\beta}{\rho}\|\boldsymbol{u}\|_{\left[L^{m+1}(\Omega)\right]^{d}}^{m+1} \leq & \frac{\mu}{\rho}\left\|K^{-1}\right\|_{L^{\infty}(\Omega)}\|\boldsymbol{u}\|_{\left[L^{\frac{m+1}{m}}(\Omega)\right]^{d}}\left\|\boldsymbol{u}_{\ell}\right\|_{\left[L^{m+1}(\Omega)\right]^{d}} \\
& +\frac{\beta}{\rho}\|\boldsymbol{u}\|_{\left[L^{m+1}(\Omega)\right]^{d}}^{m}\left\|\boldsymbol{u}_{\ell}\right\|_{\left[L^{m+1}(\Omega)\right]^{d}} \\
& +\|\boldsymbol{f}\|_{\left[L^{\frac{m+1}{m}}(\Omega)\right]^{d}}\left\|\boldsymbol{u}_{0}\right\|_{\left[L^{m+1}(\Omega)\right]^{d}}
\end{aligned}
$$

Then, for any $\epsilon>0$ and $m>1$,

$$
\frac{\mu}{\rho} \lambda_{s}\|\boldsymbol{u}\|_{L^{2}}^{2}+\frac{\beta}{\rho}\|\boldsymbol{u}\|_{\left[L^{m+1}(\Omega)\right]^{d}}^{m+1}
$$




$$
\begin{aligned}
\leq & \frac{\mu}{2 \rho}\left\|K^{-1}\right\|_{L^{\infty}(\Omega)}\left(\epsilon\|\boldsymbol{u}\|_{\left[L^{\left.\frac{m+1}{m}(\Omega)\right]^{d}}\right.}^{2}+\frac{1}{\epsilon}\left\|\boldsymbol{u}_{\ell}\right\|_{\left[L^{m+1}(\Omega)\right]^{d}}^{2}\right) \\
& +\frac{\beta}{\rho(m+1)}\left(m\|\boldsymbol{u}\|_{\left[L^{m+1}(\Omega)\right]^{d}}^{m+1}+\left\|\boldsymbol{u}_{\ell}\right\|_{\left[L^{m+1}(\Omega)\right]^{d}}^{m+1}\right) \\
& +\|\boldsymbol{f}\|_{\left[L^{\frac{m+1}{m}}(\Omega)\right]^{d}}\left\|\boldsymbol{u}_{0}\right\|_{\left[L^{m+1}(\Omega)\right]^{d}}
\end{aligned}
$$

It follows that

$$
\begin{aligned}
& \frac{\mu}{\rho} \lambda_{s}\|\boldsymbol{u}\|_{\left[L^{2}(\Omega)\right]^{d}}^{2}+\frac{\beta}{\rho}\|\boldsymbol{u}\|_{\left[L^{m+1}(\Omega)\right]^{d}}^{m+1} \\
& \leq \frac{\mu}{2 \rho}\left\|K^{-1}\right\|_{L^{\infty}(\Omega)}\left(C \epsilon\|\boldsymbol{u}\|_{\left.\left[L^{2}(\Omega)\right]^{d}\right]}^{2}+\frac{1}{\epsilon}\left\|\boldsymbol{u}_{\ell}\right\|_{\left[L^{m+1}(\Omega)\right]^{d}}^{2}\right) \\
& +\frac{\beta}{\rho(m+1)}\left(m\|\boldsymbol{u}\|_{\left[L^{m+1}(\Omega)\right]^{d}}^{m+1}+\left\|\boldsymbol{u}_{\ell}\right\|_{\left[L^{m+1}(\Omega)\right]^{d}}^{m+1}\right) \\
& +\|\boldsymbol{f}\|_{\left[L^{\frac{m+1}{m}}(\Omega)\right]^{d}}\left\|\boldsymbol{u}_{0}\right\|_{\left[L^{m+1}(\Omega)\right]^{d}}
\end{aligned}
$$

We choose $\epsilon=\frac{2 \lambda_{s}}{C\left\|K^{-1}\right\|_{L^{\infty}(\Omega)}}$ and (71) becomes

$$
\begin{aligned}
& \frac{\beta}{\rho(m+1)}\|\boldsymbol{u}\|_{\left[L^{m+1}(\Omega)\right]^{d}}^{m+1} \\
& \leq \frac{\beta}{(m+1)}\left\|\boldsymbol{u}_{\ell}\right\|_{\left[L^{m+1}(\Omega)\right]^{d}}^{m+1}+\frac{C}{4} \frac{\mu}{\rho} \frac{\left\|K^{-1}\right\|_{L^{\infty}(\Omega)}^{2}}{\lambda_{s}}\left\|\boldsymbol{u}_{\ell}\right\|_{\left[L^{m+1}(\Omega)\right]^{d}}^{2} \\
& \quad+\|\boldsymbol{f}\|_{\left[L^{\frac{m+1}{m}}(\Omega)\right]^{d}}\left\|\boldsymbol{u}_{0}\right\|_{\left[L^{m+1}(\Omega)\right] d} .
\end{aligned}
$$

Hence (63) is established.

In view of (34), we have

$$
\|\nabla p\|_{\left[L^{\frac{m+1}{m}}(\Omega)\right]^{d}} \leq\left\|\mathcal{A}\left(\boldsymbol{u}_{0}+\boldsymbol{u}_{\ell}\right)\right\|_{\left[L^{\frac{m+1}{m}}(\Omega)\right]^{d}}+\|\boldsymbol{f}\|_{\left[L^{\frac{m+1}{m}}(\Omega)\right]^{d}} .
$$

Now, applying (40) to the RHS of (73), we get

$$
\begin{aligned}
& \|\nabla p\|_{\left[L^{\frac{m+1}{m}}(\Omega)\right]^{d}} \\
& \quad \leq \frac{\mu}{\rho}\left\|K^{-1}\right\|_{L^{\infty}(\Omega)}\left\|\boldsymbol{u}_{0}+\boldsymbol{u}_{\ell}\right\|_{\left[L^{\frac{m+1}{m}}(\Omega)\right]^{d}}+\frac{\beta}{\rho}\left\|\boldsymbol{u}_{0}+\boldsymbol{u}_{\ell}\right\|_{\left[L^{m+1}(\Omega)\right]^{d}}^{m}+\|\boldsymbol{f}\|_{\left[L^{\frac{m+1}{m}}(\Omega)\right] d^{d}} .
\end{aligned}
$$

Thus (64) is established, since $\boldsymbol{u}=\boldsymbol{u}_{0}+\boldsymbol{u}_{\ell}$.

\section{Conclusion}

The well-posedness of Darcy-Forchheimer model was first established in [20]. In this paper, we extended the well-posedness results to the generalized Darcy-Forchheimer model in a two- or three-dimensional porous domain using classical arguments for nonlinear monotone saddle point problems. 
Funding

Not applicable.

\section{Abbreviations}

Not applicable

Availability of data and materials

Not applicable.

\section{Competing interests}

The authors declare that they have no competing interests.

\section{Authors' contributions}

All authors contributed equally. They both read and approved the final manuscript.

\section{Publisher's Note}

Springer Nature remains neutral with regard to jurisdictional claims in published maps and institutional affiliations.

Received: 11 June 2018 Accepted: 26 July 2018 Published online: 09 August 2018

\section{References}

1. Morales, F., Naranjo Alvarez, S.: The interaction between PDE and graphs in multiscale modeling. Opusc. Math. 37(2), 327-345 (2017)

2. Cavalcanti, M., Domingos Cavalcanti, V., Lasiecka, I., Webler, C.: Intrinsic decay rates for the energy of a nonlinear viscoelastic equation modeling the vibrations of thin rods with variable density. Adv. Nonlinear Anal. 6(2), 121-145 (2017)

3. Fairag, F.A.: Analysis and finite element approximation of a Ladyzhenskaya model for viscous flow in streamfunction form. J. Comput. Appl. Math. 206(1), 374-391 (2007)

4. Al-Smail, J.H., Messaoudi, S.A., Talahmeh, A.A.: Well-posedness and numerical study for solutions of a parabolic equation with variable-exponent nonlinearities. International Journal of Differential Equations (2018)

5. Forchheimer, P.: Wasserbewegung durch boden. Z. Ver. Dtsch. Ing. 45, 1782-1788 (1901)

6. Muskat, M.: The flow of homogeneous fluids through porous media The Mapple Press Company, York, PA

7. Firdaouss, M., Guermond, J., Le Quéré, P.: Nonlinear corrections to Darcy's law at low Reynolds numbers. J. Fluid Mech. 343, 331-350 (1997)

8. Skjetne, E., Auriault, J.L.: High-velocity laminar and turbulent flow in porous media. Transp. Porous Media 36, 131-147 (1999)

9. Ghergu, M., Radulescu, V.: Nonlinear PDEs: Mathematical Models in Biology, Chemistry and Population Genetics. Springer Science \& Business Media, New York (2011)

10. Firoozabadi, A., Katz, D.L.: An analysis of high-velocity gas flow through porous media. J. Pet. Technol. 31, 211-216 (1979)

11. Firoozabadi, A., Thomas, L.K., Todd, B.: High-velocity flow in porous media. SPE Reserv. Eng. 10, 149-152 (1995)

12. Miskimins, Jennifer, L., Lopez, H.D., Barree, R.D.: Non-Darcy flow in hydraulic fractures: does it really matter? In: SPE Annual Technical Conference and Exhibition. Society of Petroleum Engineers, (2005)

13. Aulisa, E., Bloshanskaya, L., Hoang, L.: Analysis of generalized Forchheimer equations for compressible fluids in porous media. J. Math. Phys. 50, 103102 (2009)

14. Hoang, L., Ibragimov, A.: Structural stability of generalized Forchheimer equations for compressible fluids in porous media. Nonlinearity 24, 1 (2010)

15. Douglas, J., Paes-Leme, P.J., Giorgi, T.: Generalized Forchheimer flow in porous media. Army High Performance Computing Research Center (1993)

16. Bear, J.: Dynamics of Fluids in Porous Media. Dover, New York (1988)

17. Norrier, D.H., Vries, G.D.: A survey of finite element application in fluid mechanics. In: Finite Elements in Fluid. Finite Elements in Fluids, pp. 363-395. Wiley, New York (1978)

18. Mei, C., Auriault, J.: The effect of weak inertia on flow through a porous medium. J. Fluid Mech. 222, 647-663 (1991)

19. Chauveteau, G., Thirriot, C.: (1965): Sur les pertes de charge en écoulement laminaire dans quelques géométries simple et dans le milieu poreux, IX Convegno di idraulica e costruzioni idrauliche, Trieste, III A 3

20. Girault, V., Wheeler, M.F.: Numerical discretization of a Darcy-Forchheimer model. Numer. Math. 110(2), 161-198 (2008)

21. Boffi, D., Brezzi, F., Fortin, M.: Mixed Finite Element Methods and Applications, vol. 44. Springer, Berlin (2013)

22. Brezzi, F.: On the existence, uniqueness and approximation of saddle-point problems arising from Lagrangian multipliers. RAIRO. Anal. Numér. 8(R2), 129-151 (1974)

23. Browder, F.: The solvability of non-linear functional equations. Duke Math. J. 30(4), 557-566 (1963)

24. Showalter, R.E.: Monotone Operators in Banach Space and Nonlinear Partial Differential Equations. Am. Math. Soc., Providence (2013)

25. Adams, R.A.: Sobolev spaces Academic Press, New York

26. Girault, V., Raviart, P.: Finite Element Approximation of the Navier-Stokes Equations. Springer, Berlin (1986)

27. Brezis, H.: Functional Analysis, Sobolev Spaces and Partial Differential Equations. Springer Science \& Business Media, New York (2010)

28. William, S., Martin, L.: Mean value theorem for vector valued functions. Math. Mag. 52, 157-158 (1979)

29. Rudin, W.: Principles of Mathematical Analysis. McGraw-Hill, New York (1976) 